\title{
On the Exploration and Innovation of Line Art in Animation Modeling Design
}

\author{
Yelei Chen \\ School of Art and Design Wuhan University of Technology, Wuhan 430070, China; \\ School of Art Hubei Polytechnic University, huangshi 435000, China. \\ 24394604@qq.com, vickeychanchan@163.com
}

\begin{abstract}
Keywords: Line art; material media; performance style; expression of artistic conception; innovation
\end{abstract} and practice.

\begin{abstract}
This article deals with the methods of enumerating, analysis and comparative study. It presents a way to research the linear art of animation modeling design. Here the author introduces a new approach of displaying various styles and forms of lines, which can reflect and enhance the appeal of different style of animation modeling design so as to quest for the innovation and practice in the animation modeling design. The line art of the animation modeling design is an exotic flower in the oriental arts. It does not only mean to build up the artistic quality of the line, but also to greatly improve the value of the animation modeling design. Only such an art has great vitality. This may come to a conclusion that the line art is the foundation of the animation design. The lines of the animation modeling design are not only the basis and premise of the animation, but also an inspiration for playwriting and subsequent work. The most important element of a good cartoon is a good animation modeling design. Good animation modeling design not only reflects stylistic features of the film and TV animation, can also affect the degree of interesting of the audience to the role. Further exploration of the line art would contribute to the innovation and creation of animation modeling design. In animation modeling design, the exploration and innovation of line art are often reflected in several aspects such as line shape, material media, performance style and expression of artistic conception.
\end{abstract}

\section{Introduction}

Animation itself is a kind of art. In animation modeling design, various styles and forms of lines embody the characteristics of the role. The using of lines can express true feelings. The most important element of a good cartoon is a good animation modeling design. Good animation modeling design not only reflects stylistic features of the film and TV animation, can also affect the degree of interesting of the audience to the role.

The rapid development of domestic animation leads to the boom of a series of industries. The innovation and practice of line art in modeling design can promote the development of animation art and the procession of industrial chain so as to earn more market benefits.

\subsection{The Overview of Domestic and Foreign Research.}

Line is random, variable and flexible. Line can wander in the hands of the artists and designers. The winding, the phase of the line and the line form a unique dynamic visual effect. [1] Based on the using brush, the stitch and texture, 18 kinds of line forms are concluded by Chinese ancient people, commonly known as 18 traces. [2]According to 18 traces, it already presents the artistic quality of the line, and makes use of the various styles of line to modeling. In our country's animation modeling, it embodied different line art style and diverse, such as paper cutting, shadow play, drama, ink and a variety of forms. The line has certain imagery quality and implicit beauty with very distinct Chinese characteristics. In foreign countries, Matisse once said: "the painter continues to look for the line of the desire to see where it extends, where to disappear.” The line is a way of self reaction. It can bring endless imagination and creativity to artists and designers. [3]Foreign animation design is more realistic in line art, straightforward and simple, clear. 


\section{The Form of Line Art}

Line as the transition element, compared with point, is full of dynamic and active atmosphere. Compared with the surface, the variety and rhythm of line win. [4]Artists, designers in the first place draw the characteristics of animation modeling form with the line, which is a source of design inspiration. It presented vivid modeling through bouncing lines and tension. Various types of lines bring different feelings. Horizontal line leaves the impression of the sense of balance, stability and eternity, calm. Vertical line has a towering, strong and magnificent majesty visual effect. Oblique line gives a person the rocky feeling. Broken line changes frequently. Curve makes the person feel full, smooth and soft. The combination of straight and curve greatly embodies artistic beauty.

\section{Material Medium of Line}

In animation modeling design, a variety of material medium can be used to reflect the art of lines. Different material mediums such as charcoal, pencil, needle tube pen, water-based pen, pen, mark pen, writing brush can result in different effect in performance. Lines by charcoal can show a sense of texture. Fine lies in roughness. The quantity of lighting can be denoted by charcoal line strongly. Pencil line is natural and smooth, not rough and greasy. Fine point line's beauty and tenuousness need a smooth paper to be presented. Roller-tip pen can draw stationary and fluency line. Pen line is abounding with change. Mark lines have a strong sense of adornment. Brush line requires moist paper. Lines are flexible and changeable. It can be shown as square or round, or thick or thin. Artistic line depends on the choice of the pen and paper. The varieties of paper also are many, such as rice paper, tissue paper, mulberry paper, deckle edged paper, white paper and so on. A use of different carriers can reveal the different artistic beauty of line.

\section{Performance Styles of Lines}

Artists, designers prefer to draw a lot of animation image with their own painting language and technique of expression. The animation modeling has unique performance style. The line has strong vitality, which wasn't able to be shown by the computer. A new and unique character image can constitute of the different style of line. It has strong visual feeling. Many animation masters draw the outline of animation image with use casual line ad libitum, which bring endless inspiration for animation modeling.

\subsection{Plain and Unvarnished Lines.}

The modeling design of silhouette animation "Lan Huahua" embodies the plain lines fully. It reflects the culture connotation of paper-cut in the north Shaanxi.

Plain line produces the momentum wisely, forms simple style, and does abstract transfiguration. It has both shape and spirit. There are exquisite details hidden in simple shape. There is a striking contrast between simplified contour shape and elaborately decorated character's costume. A famous fable "Be there just to make up the number" has been rearranged into a cartoon South Guo. Different painting styles fuse together. Plain style of the Han dynasty carved stones combines with portrait of the Han dynasty brick. It demonstrates magnificent strength and accurate contours. Beautifully carved lines depict dance rhythm of palace maid. The linear performance of simple building is a thick, unadorned image modeling. The emblazonment of The Palace structures has a strong sense of adornment.

\subsection{Flexible and Subtle Lines.}

Flexible and subtle lines are smooth and natural. It often has a sense of implication. Though the static lines make people feel calm, it has a dynamic visual effect. In "Havoc in Heaven”, the author uses flexible and subtle lines to present the innervations of the fairy's clothing and the rhyme of clouds. The fairy' flying posture has a sense of dynamic beauty. Wavy lines are used to reflect older features of The Jade Emperor. Subtle lines are used to draw the fairy's fingers. The figures in the Dun Huang frescoes are used for reference. 


\subsection{Interesting and Exaggerated Lines.}

In the Eastern Jin Dynasty Gu Kaizhi has done a lot of research and practice in line representation techniques. He has a painting theory of "not only for appearance, movement, gesture, shape similar". [5]Art master Wu Guanzhong also believes that "form is art itself." Art is beyond the realistic category.

Take for example "Three monks" the animation cartoon. Its modeling with smooth line is lively and interesting.

The shaping of exaggerated, humorous, vivid images is based on circular, oval, linear. This art style of animation cartoon is very typical. Because of its exaggerated entertainment modeling, "Three monks" is a classical refine, fun works.

\subsection{Resembling and Dissimilar Lines.}

Water-Color animation is the treasure of Chinese animation art. It does inherit the traditional Chinese painting ink performance technique. Its charm is shown by way of animation. It is an animation of Chinese painting art. Water-Color animation breaks the outline and boundaries of line directly. It makes use of the beauty and the effect of artificial shade. The background of freehand landscape painting fully reflected the aesthetic thought of "between resembling and dissimilar line". Resembling and dissimilar lines in the animated cartoon has a profound artistic imagery. Chinese landscape painting, figure painting and flower and bird painting are expressed incisively and vividly in great Water-Color animation such as "little tadpole looking for mommy", "the deer's bell" and "Feeling from Mountain and Water" and so on. It greatly satisfies the aesthetic needs of the audience. It completely achieved the highest state of art creation.

\section{Conclusion}

Line is a form with the most primitive and the simplest emotion and creative inspiration in any work. It is the main form of animation modeling design. Line is the soul of animations modeling design. It does not only draw tangible objects, but also performs invisible image. It is the combination of thinking in image and abstract thinking. The tempo, rhythm, balance of line and other form principle for line and the emotion complement each other. It reveals the harmony of interesting and the emotion. When designing animation model, line can conveyed effectively emotion. The priorities, thickness and shade, and proper density of line represent the art in animation design. In animation modeling design, the art of line has a strong vitality. The arrangement and composition, tempo and rhythm of the line are the key of the artistic conception. They are still the embodiment of the artists' and designers' artistic attainments.

More efforts especially the support from the society and the public are needed to promote the development of domestic animation in a real sense. The development of industry relies heavily on the attention of the consumers and the protection of the intellectual property. The practice and innovation of line art in animation modeling design is the first step to get success, which requires us to put more efforts in details and more devotion to support the development of animation industry. A newly born industry is bound to bring about rapid development as well as some system and management problems that animators should face with great bravery. Learning from some successful animation models, we should go ahead with the practice and innovation of the line art with our own characteristics in animation modeling design, in which huge economic and social benefits are implied.

\section{References}

[1] Lin lin .The Power of Line---Research the Development of Figure Painting Line from Gu Kaizhi to Li Gonglin [J]. Art \& Design, 2006(2):20 (In Chinese)

[2] Liu Yan. Line of the Meaning and Character of Modern Chinese Painting Visual Model [J]. Art Observation, 2011(2):66-69 (In Chinese) 
[3] David Rosand, Xu Bin, Wu lin, Wang Jun. Drawing Acts-Studies in Graphic Expression and Representation [M]. Shangdong Pictorial Press. 2007

[4] Wang Xiang. Line Elements of Modeling and its Application in Furniture Design [J]. Packaging Engineering, 2008(8):102-105(In Chinese) [5] Yao Yujuan. Study on the Characteristics of Line Modeling in the Plane Design [J]. Packaging Engineering, 2002(12):30-31 (In Chinese) 